\title{
Modelo Pedagógico de M-Learning em Sala de Aula Invertida (ML- SAI): Reflexões Sobre o Uso de Recursos Tecnológicos
}

Ernane Rosa Martins, IFG, Brasil, ernane.martins@ifg.edu.br Luís Manuel Borges Gouveia, UFP, Portugal, lmbg@ufp.edu.pt

Resumo. O ML-SAI é um modelo pedagógico que visa orientar atividades de mlearning, tendo como fundamentação a metodologia Ativa da Sala de Aula Invertida (SAI). Este artigo tem como objetivo relatar a aplicação do ML-SAI em cursos de nível Superior e Médio em uma instituição de ensino, com o uso dos smartphones dos próprios alunos e promover algumas reflexões sobre o uso dos recursos tecnológicos envolvidos. Para tal, foi realizada uma pesquisa exploratória, tendo como instrumento de coleta de dados: observação, questionários on-line e o registro no ambiente virtual Edmodo. Os resultados encontrados possibilitaram observar que as orientações do $M L-$ SAI permitiram uma boa integração entre os recursos tecnológicos e o ambiente escolar.

Palavras-chave: Sala de Aula Invertida, M-learning, Recursos Tecnológicos.

\section{Inverted Classroom M-Learning Model (ML-SAI): Reflections on the Use of Technology Resources}

Abstract. ML-SAI is a pedagogical model that aims to guide m-learning activities, based on the active methodology of the Inverted Classroom (IC). This article aims to report the application of ML-SAI in Higher and High School courses in an educational institution, using the students' smartphones and to promote some reflections on the use of the technological resources involved. For such, exploratory research was carried out, having as the instrument of data collection: observation, on-line questionnaires and the registration in the Edmodo virtual environment. The results showed that the ML-SAI guidelines allowed a good integration between technological resources and the school environment.

Keywords: Inverted Classroom, M-learning, Technological Resources.

\section{Introdução}

Um modelo é uma representação figurativa que reproduz a realidade de forma abstrata, esquemática e a referência. Este conceito é um dos mais importantes da atividade cientifica por permitir interpretar, comparar, simular e compreender fenômenos em diferentes situações hipotéticas. O conceito de modelo pedagógico por sua vez, foi erroneamente considerado como sinônimo de teoria de aprendizagem ou metodologia de ensino. Segundo Vendruscolo \& Behar (2016) o termo modelo pedagógico vem sendo utilizado pela comunidade científica, para designar, principalmente, estratégias e ferramentas pedagógicas, gerando uma confusão conceitual a respeito do tema. $O$ modelo pedagógico é geralmente uma reinterpretação ou simplesmente embasado por uma ou mais teorias de aprendizagem, construindo-se modelos pessoais próprios a partir destas teorias e as concepções individuais dos professores, sendo então compartilhados com os pares, buscando promover a V. $17 \mathrm{~N}^{\circ}$ 3, dezembro, 2019 RENOTE DOI: 
aprendizagem, abranger o conteúdo de ensino e desenvolver o aluno. Um modelo pedagógico é constituído por uma Arquitetura Pedagógica (AP) e as estratégias para aplicação da AP, que considera os aspectos organizacionais, instrucionais, metodológicos e tecnológicos (Behar; Passerino \& Bernardi, 2007).

A Sala de Aula Invertida (SAI), que fundamenta o modelo pedagógico ML-SAI, é uma metodologia ativa, em que o aluno realiza, em casa, algumas atividades que, normalmente, seriam realizadas na escola, e o tempo em sala de aula é reservado para a realização de atividades mais ativas, tais como: experimentos, debates, atividades em grupo, pesquisas, entre outros (Mattar, 2017). Nesta teoria os alunos assumem a responsabilidade pela sua aprendizagem, mas não significa que os professores renunciem às suas responsabilidades, e sim que passem a atuar como guias do aprendizado, fornecendo as ferramentas e estratégias necessárias para os alunos, permitindo desenvolver habilidades de autonomia, disciplina e motivação, além de aumentar o desempenho e resultados acadêmicos (Abeysekera \& Dawson, 2015).

A SAI estabelece que os alunos possam aplicar, analisar, avaliar e criar conteúdo em torno de um determinado tópico, enquanto que as tarefas de memorizar e compreender são realizadas fora da sala de aula. Ou seja, a aplicação prática do conteúdo, que é o momento mais relevante do processo de aprendizagem, é feito em sala de aula com o auxílio do professor como um guia do aprendizado (Andrade \& Chacón, 2018; Manresa, 2018). O professor tem o controle da aula, servindo como um guia e apoio para os estudantes, de forma personalizada para aprofundar certos pontos do assunto, alcançar o maior desenvolvimento e alcançar os objetivos propostos (Touchton, 2015). Estas mudanças afetam integralmente as universidades, gerando novas dúvidas e questionamentos sobre os papeis dos professores e alunos, assim como as demandas necessárias neste contexto (Thai, De Wever \& Valcke, 2017).

Akçayır \& Akçayır (2018), apresentaram uma detalhada revisão sistemática da literatura sobre o conceito da SAI, com os objetivos de examinar suas vantagens e desafios relatados por alunos e instrutores, e notar áreas potencialmente úteis de futuras pesquisas sobre o modelo invertido. A pesquisa foi realizada em 71 artigos indexados a Web of Science. As descobertas revelaram que a vantagem mais relatada da SAI é a melhoria do desempenho de aprendizagem do aluno, o que justifica a construção e experimentação de um modelo pedagógico que seja fundamentado nesta abordagem. Assim, este artigo tem como objetivo relatar a aplicação do ML-SAI e promover algumas reflexões sobre o uso dos recursos tecnológicos envolvidos. Tendo em vista o objetivo proposto, na seção 2 é apresentada a estrutura e estratégias do ML-SAI. Na seção 3 é estabelecido o método, as técnicas e procedimentos metodológicos utilizados. Na seção 4, apresenta-se os resultados encontrados. Na seção 5 temos algumas reflexões a respeito da experimentação realizada. Por fim, a seção 6 apresenta algumas considerações sobre o presente trabalho.

\section{Estrutura e Estratégias do ML-SAI}

O modelo pedagógico ML-SAI foi construído a partir de três estudos de caso exploratórios preliminares, em conjunto com a revisão bibliográfica realizada. Utilizando os conceitos da SAI combinados com as tecnologias móveis: Kahoot, WhatsApp e Facebook, investigando as potencialidades da utilização da SAI com o auxílio de tecnologias móveis. Assim, os resultados encontrados nestes estudos preliminares, são apresentados na Tabela 1. 
Tabela 1 - Resultados dos estudos preliminares que fundamentaram o modelo pedagógico ML-SAI.

\begin{tabular}{l}
\hline Resultados encontrados nos estudos preliminares \\
\hline As limitações e dificuldades de ordem financeira e técnica podem excluir alguns alunos que não dispõem \\
de smartphones, planos de Internet em seus celulares ou mesmo Internet em suas residências, dificultando \\
o uso e principalmente o acesso dos alunos às ferramentas e aos recursos digitais disponíveis; \\
\hline Benefícios significativos, como: baixo custo, acessibilidade, interatividade e aprendizagem colaborativa; \\
\hline Mediação do professor como fator fundamental, propondo temas e estimulando a participação dos alunos, \\
identificando o contexto da sala de aula, dos alunos e da turma, estabelecendo regras e normas para \\
utilização dos dispositivos móveis, deixando claro os objetivos e os motivos das atividades propostas, \\
verificando as limitações relevantes e os recursos tecnológicos necessários que serão utilizados, assim \\
como, os papeis dos professores e dos alunos neste processo; \\
\hline Confirmação de que é fundamental um planejamento bem estruturado por parte do professor.
\end{tabular}

O modelo pedagógico ML-SAI foi formatado para fornecer algumas sugestões de estratégias a professores interessados em utiliza-lo, orientando estes no desenvolvimento das atividades de m-learning. No ML-SAI a AP foi reestruturada e fundamentada levando em consideração os conceitos da SAI, os aspectos relacionados a utilização dos dispositivos móveis e os estudos exploratórios preliminares realizados, assim, a AP foi estabelecida em seis aspectos, sendo estes: contexto, normatização, papeis, tecnologias, ações e limitações. A AP e as estratégias para a Aplicação da AP definidas para as atividades de m-learning estão apresentadas na Tabela 2.

Tabela 2 - Modelo pedagógico ML-SAI (Martins \& Gouveia, 2019).

\begin{tabular}{|c|c|}
\hline $\mathbf{A P}$ & Estratégias para a Aplicação da AP \\
\hline Contexto & $\begin{array}{l}\text { Definir os objetivos e motivos das atividades e ações proposta, deixando-os claros para todos } \\
\text { os envolvidos; } \\
\text { Identificar os instrumentos, recursos, características das atividades e ações, dos alunos e do } \\
\text { curso; }\end{array}$ \\
\hline Norma & $\begin{array}{l}\text { Organizar regras e procedimentos para orientar as ações e interações; } \\
\text { Estabelecer normas para utilização dos dispositivos móveis (quando utilizar, qual a } \\
\text { finalidade, etc.); }\end{array}$ \\
\hline Papeis & $\begin{array}{l}\text { Compreender o papel do aluno no processo de aprendizagem, suas motivações, interesses e } \\
\text { habilidades; } \\
\text { Entender o papel do professor como condutor e facilitador da aprendizagem; }\end{array}$ \\
\hline Tecnologias & $\begin{array}{l}\text { Definir os dispositivos móveis, aplicativos e recursos tecnológicos que serão utilizados, } \\
\text { considerando as características físicas, técnicas e funcionais dos mesmos, tais como: } \\
\text { ambiente virtual, Sílabe, Moodle, Facebook, Khan Academy, YouTube, vídeo-aula, músicas, } \\
\text { slides, fotografias, áudios, textos, entre outros, estabelecendo prioridade para aplicativos } \\
\text { livres e gratuitos; } \\
\text { Verificar a necessidade e disponibilidade de conexão com a Internet; }\end{array}$ \\
\hline Ações & $\begin{array}{l}\text { Especificar se as ações serão individuais, em grupo ou ambas, se estas serão comuns a todos } \\
\text { os alunos ou diferenciadas por aluno ou grupo de alunos; } \\
\text { Definir ferramentas de comunicação e sistemas de apoio para dar suporte aos alunos em caso } \\
\text { de dificuldades; } \\
\text { Definir se as ações serão realizadas em um mesmo local, ao mesmo tempo ou em locais e } \\
\text { momentos distintos; } \\
\text { Estabelecer práticas educacionais favoráveis ao aprendizado (situações problemas, } \\
\text { aplicações práticas, colaborativas, autônomas, críticas, em contextos reais, pesquisas), } \\
\text { levando em consideração os ambientes de aprendizagem (on-line, salas de aula, laboratórios) } \\
\text { de preferência com os dispositivos móveis dos próprios alunos; } \\
\text { Incentivar a interação entre os alunos e com o professor, por meio do uso de dispositivos } \\
\text { móveis, com foco no desenvolvimento da atividade proposta; } \\
\text { Determinar os mecanismos de avaliação de desempenho e certificação da aprendizagem, se } \\
\text { individuais ou em equipes, de preferência continua, e disponibiliza-los para os alunos; } \\
\text { Estabelecer momentos de reflexão e análise das atividades realizadas, buscando colaborar na }\end{array}$ \\
\hline
\end{tabular}

V. $17 \mathrm{~N}^{\mathrm{o}}$ 3, dezembro, 2019

DOI: 
melhoria continua de novas atividades;

Estruturar os conteúdos que serão disponibilizados em ambiente virtual, para que os alunos possam acessa-los por meio de um dispositivo móvel, quando e quantas vezes quiserem, se possível com o acompanhamento das visualizações pelo professor;

Realizar uma curadoria dos conteúdos já existentes na Internet, por meio de plataformas como Khan Academy e o YouTube em busca de bons vídeos educativos, ou caso não sejam encontrados, gravar vídeos ou áudios utilizando as ferramentas que existem no próprio dispositivo móvel;

Estimular diferentes formas de aprendizado por meio de diferentes fontes de conteúdo, tais como: vídeos, áudios, imagens, textos, slides, questões, entre outras;

Elaborar um roteiro de atividades do que será feito dentro da sala de aula, de modo a otimizar o tempo em sala de aula, utilizando projetos, trabalhos ou solução de problemas, que se conectem com o que foi visto previamente na plataforma;

Levantar os principais pré-requisitos das atividades e possíveis distratores do aprendizado; Identificar quais conteúdos podem ser melhor trabalhados com tecnologias móveis;

Verificar se os materiais pedagógicos podem ser utilizados em dispositivos móveis, Limitações considerando tamanho da tela, usabilidade, capacidade de armazenamento e modelos de dispositivos diferentes;

Verificar a disponibilidade de dispositivos móveis, tomadas para recarregar as baterias dos celulares, conexão com a Internet, quando necessário, e se os aplicativos apresentam interface adequada a aprendizagem do conteúdo.

O ML-SAI foi concebido para colaborar com a realização de diversas atividades de m-learning, com diferentes conteúdos em dispositivos móveis.

\section{Metodologia}

Utilizou-se o método de estudo de caso, por ser um estudo de natureza empírica que investiga um determinado fenômeno, geralmente contemporâneo, dentro de um contexto real de vida, tratando-se de uma análise aprofundada de um ou mais objetos (casos), permitindo seu amplo e detalhado conhecimento (Gil, 2002). O seu objetivo é aprofundar o conhecimento acerca de um problema não suficientemente definido, visando estimular a compreensão, sugerir hipóteses e questões ou simplesmente desenvolver a teoria. A presente pesquisa é classificada como exploratória, de cunho quantitativa e qualitativa, pois visa desenvolver, esclarecer ou modificar conceitos e ideias sobre determinado assunto (Gil, 1999). O desenvolvimento dessas estratégias teve como instrumentos de coleta de dados a observação realizada pelo pesquisador de forma não estruturada, a percepções dos alunos e professores envolvidos, recolhidas por meio do questionário on-line e ainda mediante os registros do ambiente virtual Edmodo. A observação teve foco no uso dos recursos tecnológicos dentro da proposta.

O ML-SAI foi utilizado nas atividades elaboradas pelos professores durante o primeiro semestre de 2019, no curso superior noturno de Sistemas de Informação (SI), com a participação de 90 alunos, e no curso de nível médio técnico em Informática para a Internet, com a participação de 45 alunos, ambos cursos presenciais de uma instituição de ensino, a escolha pelos cursos visou comparar os resultados encontrados em níveis de ensino diferentes, no caso, Ensino Médio e Superior. Os dispositivos móveis adotados foram os celulares dos próprios alunos, para permitir aproximação com o que os alunos estão habituados a utilizar em seu dia a dia e facilitar a aprendizagem. O ambiente de aprendizagem on-line utilizado foi o Edmodo, escolhido por ser o que os alunos utilizavam nas aulas e por estar disponível para acesso por aplicativo de smartphones.

As questões que integraram o questionário foram: Os participantes possuíam smartphone, qual o tipo e recursos dos mesmos? Os participantes utilizavam o smartphone para estudo? Os dispositivos móveis colaboram para fins educativos? O uso V. $17 \mathrm{~N}^{\circ}$ 3, dezembro, 2019 RENOTE DOI: 
de diversos recursos tecnológicos no apoio à disciplina contribuiu para a aprendizagem? Os smartphones foram recursos importantes para a aprendizagem? Os aplicativos do smartphone utilizados foram fáceis de usar? A proposta de uso do smartphone, foi importante para a disciplina? Foram utilizados recursos tecnológicos adequados? Apresente alguns comentários que considere relevante? A análise crítica dos dados recolhidos no questionário aplicado aos alunos, ocorreu organizando, cruzando e comparando as informações similares por contextos. A metodológica foi apoiada por recursos tecnológicos digitais, tais como: YouTube, WhatsApp, Edmodo, entre outros.

\section{Resultados}

Exemplificando alguns dos projetos realizados, temos em um deles a elaboração de um programa de cálculo de matrizes, que realizava as quatro operações básicas da matemática: soma, subtração, multiplicação e divisão de matrizes. Os alunos aprenderam as estruturas das matrizes conforme a linguagem de programação por meio de vídeo antes das aulas e identificaram as possíveis soluções para o problema em sala de aula, todos os alunos participantes criaram alguma implementação, ainda que parcial para o problema, mas pelo aprendizado e o desenvolvimento do trabalho ser praticamente sem a ajuda do professor, os resultados foram considerados impressionantes pelos alunos e professor. Os alunos foram orientados a pensar em um problema real, o que despertou o interesse dos alunos, visando disponibilizar está aplicação posteriormente para a realização deste tipo de cálculo. Mesmo sendo um projeto simples e inicial, a intenção foi motivar os alunos a realizarem algo útil, assim adotou-se sempre esta mesma estratégia nos demais projetos.

Um segundo exemplo de projeto realizado foi a implementação de uma calculadora de massa corporal, onde os alunos precisaram pesquisar como determinar a massa corporal de homens e mulheres utilizando os seus smartphones, que antes eram utilizados para atividades sem importância, tornando-os instrumentos imprescindíveis para realizar as pesquisar e facilitar o aprendizado. Apesar dos alunos relatarem que precisaram dedicar mais tempo para a realização dos projetos, reconheceram que os benefícios foram evidentes em termos de aprendizagem do conteúdo e crescimento pessoal.

Quando perguntados se os participantes possuíam smartphone, qual o tipo e recursos dos mesmos? Todos os alunos participantes afirmaram possuir smartphone, com acesso à Internet e aplicativos digitais. Nesta pergunta pretendia-se saber quantos em cada turma possuía smartphone. Observou-se que outros aparelhos móveis, como palmtop e $m p 3$, não são utilizados pelos estudantes investigados. Em relação a questão sobre qual smartphone os alunos possuíam, pretendia-se saber se algum tipo de smartphone compromete a utilização do ML-SAI. Os estudantes relataram que utilizam diversos tipos de aparelhos, tais como: Moto G5, iPhone 6s, Galaxy Gran Prime, IPhone 8 Plus, Samsung J2 prime, Galaxy j5, iPhone 6, Samsung J5 prime, Samsung J7 pro. Sendo que todos eles apresentaram plenas condições técnicas de serem utilizados junto ao ML-SAI, demostrando que os alunos geralmente possuem aparelhos modernos, que podem ser amplamente utilizados nos meios educacionais. Relativo a pergunta sobre os recursos dos aparelhos utilizados, pretendia-se saber quais os recursos do smartphone favorecem a utilização do modelo pedagógico ML-SAI. Entre os principais recursos descritos estão: acesso à Internet; leitor de arquivos de slides, vídeos, PDF e aplicativos digitais. Alguns alunos disseram que utilizaram ainda recursos como envio de SMS e Bluetooth. 
Os participantes utilizavam o smartphone para estudo? Nesta pergunta pretendia-se saber como os estudantes utilizam seu smartphone no estudo e se isto é significativo de alguma forma para eles. Os alunos investigados afirmaram que estão familiarizados com a utilização do smartphone na educação, e já utilizaram os mesmos em diversas disciplinas em seus cursos e de várias formas possíveis, tais como: com a utilização de vídeo aulas, pesquisas textuais, vídeos do YouTube, slides das disciplinas, leituras de PDF, ambiente virtual Edmodo e sites de busca. Os dispositivos móveis colaboram para fins educativos? Nesta pergunta pretendia-se saber a opinião dos alunos em relação ao uso dos dispositivos móveis para fins educativos. Todos os participantes afirmaram que os dispositivos móveis quando utilizados no ensino podem colaborar na superação das dificuldades de aprendizagem, principalmente por facilitar o acesso aos materiais educacionais e a comunicação entre os indivíduos envolvidos. O uso de diversos recursos tecnológicos no apoio à disciplina contribuiu para a aprendizagem? Nesta pergunta pretendia-se saber a opinião dos alunos em relação aos recursos tecnológicos como apoio à disciplina. Os percentuais apresentados na Tabela 3 mostram que, na opinião da maioria dos respondentes, a proposta de uso de diversos recursos tecnológicos colaborou efetivamente para o aprendizado.

Tabela 3 - Recursos tecnológicos foram apoio para a aprendizagem.

\begin{tabular}{llllll}
\hline Opções & $\begin{array}{l}\text { Concordo } \\
\text { plenamente }\end{array}$ & Concordo & $\begin{array}{l}\text { Não concordo nem } \\
\text { discordo }\end{array}$ & Discordo & $\begin{array}{l}\text { Discordo } \\
\text { completamente }\end{array}$ \\
\hline Míveis & $70 \%$ & $30 \%$ & $0 \%$ & $0 \%$ & $0 \%$ \\
\hline Superior & $48 \%$ & $33 \%$ & $15 \%$ & $4 \%$ & $0 \%$ \\
\hline Geral & $57 \%$ & $32 \%$ & $9 \%$ & $2 \%$ & $0 \%$ \\
\hline
\end{tabular}

A utilização de diversos recursos tecnológicos foi amplamente elogiada pelos alunos, por facilitar a assimilação dos conteúdos, principalmente os assuntos que apresentam geralmente maior dificuldade de aprendizado. Os smartphones foram recursos importantes para a aprendizagem? Nesta pergunta pretendia-se saber a opinião dos alunos em relação a importância dos smartphones na aprendizagem. Os percentuais apresentados na Tabela 4 mostram que, na opinião da maioria dos respondentes, os smartphones foram recursos importantes para o aprendizado.

Tabela 4 - Smartphones foram importantes na aprendizagem.

\begin{tabular}{llllll}
\hline Opções & $\begin{array}{l}\text { Concordo } \\
\text { plenamente }\end{array}$ & Concordo & $\begin{array}{l}\text { Não concordo nem } \\
\text { discordo }\end{array}$ & Discordo & $\begin{array}{l}\text { Discordo } \\
\text { completamente }\end{array}$ \\
\hline Míveis & $20 \%$ & $60 \%$ & $20 \%$ & $0 \%$ & $0 \%$ \\
\hline Superior & $33 \%$ & $47 \%$ & $20 \%$ & $0 \%$ & $0 \%$ \\
\hline Geral & $28 \%$ & $52 \%$ & $20 \%$ & $0 \%$ & $0 \%$ \\
\hline
\end{tabular}

$\mathrm{O}$ apoio constante do professor nas atividades on-line, principalmente pelo uso dos smartphones e aplicativos de mensagens instantâneas, possibilitaram a retirada de dúvidas no momento de primeiro contato com o conteúdo, o que foi elogiada por todos os envolvidos. Os aplicativos do smartphone utilizados foram fáceis de usar? Nesta pergunta pretendia-se saber a opinião dos alunos em relação a usabilidade dos aplicativos dos smartphones. Os percentuais apresentados na Tabela 5 mostram que, na opinião da maioria dos respondentes, os aplicativos dos smartphones foram extremamente fáceis de usar, principalmente pelo fato dos alunos já estão familiarizados com os aplicativos de mensagens e redes sociais no seu dia a dia, e mesmo nos aplicativos educativo, os alunos apresentam grande facilidade de aprender a utiliza-los. 
Tabela 5 - Aplicativos foram fáceis de usar.

\begin{tabular}{llllll}
\hline Opções & $\begin{array}{l}\text { Concordo } \\
\text { plenamente }\end{array}$ & Concordo & $\begin{array}{l}\text { Não concordo nem } \\
\text { discordo }\end{array}$ & Discordo & $\begin{array}{l}\text { Discordo } \\
\text { completamente }\end{array}$ \\
\hline Níveis & $40 \%$ & $60 \%$ & $0 \%$ & $0 \%$ & $0 \%$ \\
\hline Médio & $42 \%$ & $40 \%$ & $16 \%$ & $2 \%$ & $0 \%$ \\
\hline Superior & $42 \%$ & $48 \%$ & $10 \%$ & $1 \%$ & $0 \%$ \\
\hline Geral & $41 \%$ & & & \\
\hline
\end{tabular}

É bom sempre alertar que o uso dos aplicativos dos smartphones requerem certos cuidados, precisando estabelecer que o ambiente on-line também é um espaço escolar, que necessitam de orientações para o uso pedagógico adequado. A proposta de uso do smartphone, foi importante para a disciplina? Nesta pergunta pretendia-se saber a opinião dos alunos em relação ao uso dos smartphones nas disciplinas. Os percentuais apresentados na Tabela 6 mostram que, na opinião da maioria dos respondentes, a proposta do uso do smartphone foi importante para a disciplina.

Tabela 6 - Uso dos smartphones foi importante.

\begin{tabular}{llllll}
\hline Opções & $\begin{array}{l}\text { Concordo } \\
\text { plenamente }\end{array}$ & Concordo & $\begin{array}{l}\text { Não concordo nem } \\
\text { discordo }\end{array}$ & Discordo & $\begin{array}{l}\text { Discordo } \\
\text { completamente }\end{array}$ \\
\hline Níveis & $10 \%$ & $70 \%$ & $20 \%$ & $0 \%$ & $0 \%$ \\
\hline Médio & $42 \%$ & $14 \%$ & $4 \%$ & $0 \%$ \\
\hline Geral & $28 \%$ & $54 \%$ & $16 \%$ & $2 \%$ & $0 \%$ \\
\hline
\end{tabular}

Alguns alunos relataram que a utilização do smartphone ajudou na compreensão dos conteúdos da disciplina, pois muitos trabalham e estudam, não tendo tempo livre para acessar a Internet, mas por meio do smartphone podem acessar nos momentos de folga. A maioria dos alunos afirmaram que a utilização do smartphone favoreceu tanto o aprendizado personalizado, através de mensagens instantâneas e alertas, quanto o aprendizado colaborativo, por meio de salas de bate-papo e quadros de discussões, provocando reflexão, comunicação e cooperação. Comprovou-se que os smartphones quando adotados corretamente são excelentes ferramentas de apoio ao aprendizado. Foram utilizados recursos tecnológicos adequados? Nesta pergunta pretendia-se saber na opinião dos alunos se os recursos tecnológicos foram adequados. Os percentuais apresentados na Tabela 7 demostram que, na opinião da maioria dos respondentes, os recursos tecnológicos utilizados foram adequados dentro dos objetivos propostos.

Tabela 7 - Os recursos tecnológicos foram adequados.

\begin{tabular}{|c|c|c|c|c|c|}
\hline $\begin{array}{l}\text { Opções } \\
\text { Níveis }\end{array}$ & $\begin{array}{l}\text { Concordo } \\
\text { plenamente }\end{array}$ & Concordo & Não concordo nem & Discordo & Discordo \\
\hline Médio & $30 \%$ & $40 \%$ & $30 \%$ & $0 \%$ & $0 \%$ \\
\hline Superior & $47 \%$ & $40 \%$ & $13 \%$ & $0 \%$ & $0 \%$ \\
\hline Geral & $40 \%$ & $40 \%$ & $20 \%$ & $0 \%$ & $0 \%$ \\
\hline
\end{tabular}

A maioria dos alunos, afirmaram que os recursos tecnológicos utilizados, auxiliaram na execução das tarefas, tais como: nas anotações das ideias, na comunicação entre os alunos, na consulta de informações via Internet, no registro de fatos através das câmeras digitais e gravação de áudios. Os recursos tecnológicos utilizados aumentaram as possibilidades de acesso aos conteúdos didáticos, podendo acessa-los em qualquer lugar e a qualquer momento, de acordo com a conectividade do dispositivo, expandiram as possibilidades de estratégias de aprendizado disponíveis para serem utilizadas, fornecendo meios para o desenvolvimento de métodos inovadores de ensino, utilizando os recursos disponíveis da computação e da mobilidade. Apresente alguns comentários? Nesta pergunta pretendia-se saber as opiniões gerais dos alunos de forma livre sobre a utilização do modelo pedagógico ML-SAI. Observa-se que os alunos demostraram uma V. $17 \mathrm{~N}^{\circ}$ 3, dezembro, 2019 RENOTE DOI: 
excelente receptividade em relação ao ML-SAI. Algumas destas justificativas foram: "Acredito que os avanço das novas tecnologias e sua popularização devem ser inseridas na educação para um melhor aproveitamento"; "Os métodos adotados foram bem interessantes, achei bem legal a disponibilização do material na web, funcionou"; "O uso do smartphone fez toda a diferença, contribuindo para o aprendizado, pois muitos estudam e trabalham, podendo acessar o conteúdo nos momentos de folga".

\section{Discussões}

Visando aprofundar as reflexões sobre o uso pedagógico do smartphone e o uso de recursos tecnológicos com o ML-SAI, forma realizadas algumas comparações com outros trabalhos encontrados na literatura. Assim, um trabalho que explorou a mesma temática foi o estudo de Rockembach \& Garré (2018) em que apresentaram um estudo de caso que experimentou o uso pedagógico de tecnologias móveis por meio do uso do aplicativo WhatsApp combinado com a utilização dos princípios da SAI, buscando intensificar as discussões e reflexões acerca do conteúdo da disciplina de Química, com a participação de jovens entre 14 e 16 anos. Neste foi possível observar as inúmeras possibilidades de exploração do uso pedagógico do smartphone. Apresentando aspectos positivos na aproximação dos estudantes com o conteúdo estudado. Para os autores, as aulas tradicionais eram propicias a uma constante falta de interesse dos alunos pelas aulas e com muita distração por outras coisas, geralmente o próprio smartphone dos alunos, levando as escolas a proibirem o uso de dispositivos eletrônicos em sala de aula, promovendo um distanciamento entre os alunos e a escola. Infelizmente somente trazer elementos tecnológicos para a escola não garantem o interesse dos alunos e o sucesso das práticas escolares, elas precisam, estar acompanhadas de diferentes formas de interação entre os estudantes e os conteúdos. As práticas realizadas por estes autores confirmaram os resultados encontrados neste presente trabalho, pois proporcionou momentos importantes de participação dos alunos em sala de aula, demonstrando que os estudantes haviam se ocupado em pesquisar acerca do tema trabalhado no grupo de discussões antes das aulas, evidenciando o potencial da proposta de utilizar os conceitos da SAI combinada com o uso de aplicativos dos smartphones.

Ficou evidente a partir dos resultados encontrados que a maioria dos alunos estão expostos e familiarizados com o uso das interfaces e recursos tecnológicos, em especial com o smartphone, provavelmente por serem usuários frequentes das tecnologias digitais, conhecendo e dominando as suas funcionalidades. Confirmando o pensamento de Prensky (2012), de que a tecnologia pode ser uma aliada importante para os alunos em termos educacionais, geralmente pelo fato de estarem cada vez mais presente em seu cotidiano. Moran $(2012$, p.9) afirma que para os jovens, o espaço físico e o virtual estão integrados, completam-se e combinam-se em uma só interação. Um ponto importante, que precisa ser observado é a necessidade de adaptação da forma de apresentação, quantidade de atividades e conteúdos disponibilizados, levando em consideração a interface digital escolhida e o objetivo de ensino, identificando as características especificas de cada aluno. A escolha de determinada abordagem, não garantirá eficiência e sucesso para a aula, é importante a escolha das melhores ações possíveis para atender cada aluno de forma individual, revendo, adaptando e atualizando as estratégias de aprendizagem, de modo a tirar o máximo de proveito das tecnologias digitais dentro do contexto de ensino, sempre em parceria com o aluno. $\mathrm{O}$ uso de recursos tecnológicos como auxílio pedagógico requer certos cuidados, para garantir o cumprimento dos objetivos determinados e o entendimento dos conteúdos. Este estudo 
confirma o trabalho de Araújo (2018) que afirma não existir um manual pronto e definitivo sobre como usar as tecnologias para fins educacionais, pois seu uso transcende o técnico e o instrumental. Sendo necessário pensar as tecnologias como interfaces integradoras e funcionais para a formação humana, que quando bem orientadas podem trazer múltiplas vantagens ao processo educacional.

Assim como no trabalho de Modelski et al. (2019), este estudo também demostra que os momentos de reflexões e trocas de experiências sobre os recursos tecnológicos vivenciados pelos docentes são de extrema importância, pela possibilidade de desenvolvimento de novas concepções pedagógicas relacionadas ao uso destes em sala de aula. O documento da Unesco (2014, p. 18) sobre aprendizagem utilizando recursos tecnológicos móveis, afirma que o uso destes, pode auxiliar os professores a usar o tempo de aula de forma mais efetiva, utilizando tecnologias móveis para tarefas passivas, como ouvir ou assistir uma aula expositiva em casa, e terem mais tempo para discutir ideias, compartilhar interpretações alternativas, trabalhar em grupo e participar de atividades de laboratório, na escola. O que está perfeitamente alinhado com o MLSAI, com a implementação de intervenções pedagógicas baseadas na adoção das abordagens da SAI, combinadas com tecnologias educacionais móveis. O que vai de encontro ainda ao trabalho de Sánchez-Rivas et al. (2019) que afirmam que a SAI e os dispositivos móveis formam um binômio (modelo pedagógico e recurso tecnológicos). Munhoz (2015) diz que a SAI permite a integração perfeita das tecnologias no ambiente escolar, permitindo a disponibilização e acesso de diversos conteúdos e explicações por meio de vários recursos, tais como: fórum, chats, hiperlinks, vídeo aula, tutoriais, entre outros. Sobre a utilização dos recursos tecnológicos Bergmann \& Sams (2016, p. 18) dizem que "A inversão fala a língua dos estudantes de hoje", pela infinidade de recursos digitais disponíveis e que podem ser utilizados nesta metodologia ativa.

\section{Considerações Finais}

Este artigo teve como objetivo relatar a aplicação do ML-SAI em cursos de nível Superior e Médio de uma instituição de ensino, com o uso dos smartphones dos alunos e promover algumas reflexões sobre o uso dos recursos tecnológicos envolvidos. Analisando a aplicação do ML-SAI, foi possível observar, que as orientações do MLSAI permitiram uma boa integração entre os recursos tecnológicos e o ambiente escolar, principalmente devido as características inerentes aos alunos e aos recursos digitais disponíveis atualmente. Apresentando ampla possibilidade do ML-SAI contribuir com orientações para o planejamento e a realização de ações e práticas pedagógicas de $m$ learning por professores de diferentes áreas. O ML-SAI apresentou uma boa aceitação entre os alunos e professores, auxiliou no planejamento e execução de abordagens pedagógicas, envolveu as tecnologias na educação, aliou as vantagens do digital ao presencial e melhorou o aproveitamento dos recursos tecnológicos. Limitando-se a um estudo de caso, o que impossibilita a generalização dos resultados. Pretende-se realizar novas experimentações mais abrangentes, de modo a validar e consolidar o ML-SAI, e disponibiliza-lo em forma digital para divulga-lo, possibilitando a sua ampla utilização.

\section{Referências Bibliográficas}

ABEYSEKERA, L.; DAWSON, P. Motivation and cognitive load in the flipped classroom: definition, rationale and a call for research. Higher Education Research \& Development, v. 34, n. 1, p. 1-14, 2015. 
AKÇAYIR, G; AKÇAYIR, M. The flipped classroom: A review of its advantages and challenges. Computers \& Education, v. 126, p. 334-345, 2018.

ANDRADE, E.; CHACÓN, E. Implicaciones teóricas y procedimentales de la clase invertida. Pulso. Revista de Educación, n. 41, p. 251-267, 2018.

ARAÚJO, M. DE S. Ensino-aprendizagem com tecnologias digitais na formação inicial de professores de inglês. Trabalhos em Linguística Aplicada, v. 57, n. 3, p. 1590-1614, 2018.

BEHAR, P. A.; PASSERINO, L.; BERNARDI, M. Modelos Pedagógicos para Educação a Distância: pressupostos teóricos para a construção de objetos de aprendizagem. RENOTE Revista Novas Tecnologias na Educação, v. 5, nº 2, p. 1-10, 2007.

BERGMANN, J.; SAMS, A. Sala de Aula Invertida: uma metodologia ativa de aprendizagem. Rio de Janeiro: LTC, 114 p, 2016.

GIL, A. C. Como elaborar projetos de pesquisa. 4. Ed. São Paulo: Atlas, 2002.

GIL, A. C. Métodos e técnicas de pesquisa social. São Paulo: Atlas, 1999.

MANRESA, S. T. Flipped Classroom: Un modelo pedagógico eficaz en el aprendizaje de Science. Revista Iberoamericana de Educación, v. 76, n. 1, p. 9-22, 2018.

MARTINS, E. R.; GOUVEIA, L. M. B. M-Learning e Sala de Aula Invertida: Construção de um Modelo Pedagógico (ML-SAI). In: Solange Aparecida de Souza Monteiro. (Org.). Inquietações e proposituras na formação docente. 1ed. Ponta Grossa, PR: Atena Editora, v. 1, p. 184-192, 2019.

MATTAR, J. Metodologias Ativas: para a educação presencial, blended e a distância. 1 ed. São Paulo: Artesanato Educacional, 118p, 2017.

MODELSKI, D.; GIRAFFA, L. M. M.; CASARTELLI, A. DE O. Tecnologias digitais, formação docente e práticas pedagógicas. Educação e Pesquisa, São Paulo, v. 45, p. 1-17, 2019.

MORAN, J. M. A Educação que Desejamos: Novos desafios e como chegar lá. $5^{\mathrm{a}}$ ed. Campinas, São Paulo: Papirus, 2012.

MUNHOZ, A. S. Vamos inverter a sala de aula? 1 ed, Clube de Autores, 150 p, 2015.

PRENSKY, M. From digital natives to digital wisdom: hopeful essays for 21 st century learning. California: Corwin, a Sage Company, 2012.

ROCKEMBACH, G. R.; GARRÉ, B. H. O WhatsApp e os novos modos de aprender dos Jovens na atualidade. Revista Thema, v. 15, n. 4, p. 1404-1413, 2018.

SÁNCHEZ-RIVAS, E.; SÁNCHEZ-RODRÍGUEZ, J.; RUIZ-PALMERO, J. Percepción del alumnado universitario respecto al modelo pedagógico de clase invertida. Magis, Revista Internacional de Investigación en Educación, v. 11, n. 23, p. 151-168, 2019.

THAI, N.T.T., DE WEVER, B.; VALCKE, M. The impact of a flipped classroom design on learning performance in higher education: Looking for the best "blend" of lectures and guiding questions with feedback. Computers \& Education, v. 107, p. 113-126, 2017.

TOUCHTON, M. Flipping the Classroom and Student Performance in Advanced Statistics: Evidence from a Quasi-Experiment. Journal of Political Science Education, v.11, n. 1, p. 28-44, 2015.

UNESCO. Diretrizes de Políticas Para a Aprendizagem Móvel. 2014. Disponível em: http://www.bibl.ita.br/UNESCO-Diretrizes.pdf. Acesso em: 19 de setembro de 2019.

VENDRUSCOLO, M. I.; BEHAR, P. A. Investigando modelos pedagógicos para educação a distância: desafios e aspectos emergentes. Educação, v. 39, n. 3, p. 302-311, 2016.

V. $17 \mathrm{~N}^{\circ}$ 3, dezembro, 2019

RENOTE

DOI: 